\title{
Design of a $90^{\circ}$ Overmoded Waveguide Bend*
}

\author{
C. Nantista ${ }^{\circ}$, N.M. Kroll ${ }^{\ddagger}$ and E.M. Nelson \\ Stanford Linear Accelerator Center \\ Stanford University, Stanford, CA 94309 USA
}

\section{Abstract}

A design for a $90^{\circ}$ bend for the $\mathrm{TE}_{01}$ mode in overmoded circular waveguide is presented. A pair of septa, symmetrically placed perpendicular to the plane of the bend, are adiabatically introduced into the waveguide before the bend and removed after it. Introduction of the curvature excites five propagating modes in the curved section. The finite element field solver YAP is used to calculate the propagation constants of these modes in the bend, and the guide diameter, septum depth, septum thickness, and bend radius are set so that the phase advances of all five modes through the bend are equal modulo $2 \pi$. To a good approximation these modes are expected to recombine to form a pure mode at the end of the bend.

\section{INTRODUCTION}

Some designs for the Next Linear Collider[1] (NLC) transmit power from the source (a klystron or the output of a pulse compressor) to the accelerator structure in the $\mathrm{TE}_{01}$ mode of overmoded circular waveguide in order to have small transmission loss. The waveguide run from the source to the accelerator includes some $90^{\circ}$ bends. Ideally these bends would be loss-less.

Two algorithms and some results are presented for the design of one type of overmoded waveguide bend. A curved section of waveguide connects two straight sections as shown in Figure 1a. The curvature in the bend is constant so the waveguide follows a $90^{\circ}$ arc with radius of curvature $\rho_{c}$ between the two straight sections. The crosssection of the waveguide is uniform throughout the curved section, but the cross-section is not simply a circle. The cross-section and radius of curvature $\rho_{c}$ will be chosen so that the incoming wave propagates through the curved section with negligible mode conversion. This is the principal form of loss considered here. Reflection and wall losses are only considered heuristically. The straight sections are adiabatic tapers from and to circular waveguide.

\section{TELEGRAPHIST'S EQUATION}

Curvature in overmoded waveguide causes coupling between the straight guide modes. Such coupling is afforded by the generalized telegraphist's equations[2], which have been applied to curved circular guide[3]. In terms of the forward and backward wave amplitudes, $a_{n}^{ \pm}$, these are

$$
\frac{d a_{m}^{ \pm}}{d z}=\mp i \sum_{n}\left(C_{m n}^{ \pm} a_{n}^{+}+C_{m n}^{\mp} a_{n}^{-}\right),
$$

\footnotetext{
* Work supported by U.S. Department of Energy contract DE-AC03-76SF00515 and grants

DE-FG03-92ER40759 and DE-FG03-92ER40695.

$\diamond$ Visitor from Department of Physics, UCLA, Los Angeles, CA 90024.

$\ddagger$ Also from Department of Physics, UCSD, La Jolla, CA 92093.
}

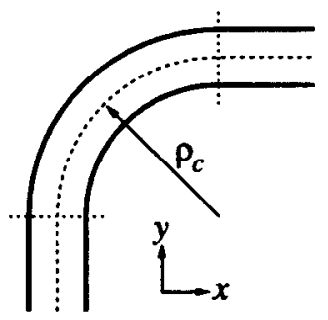

(a)

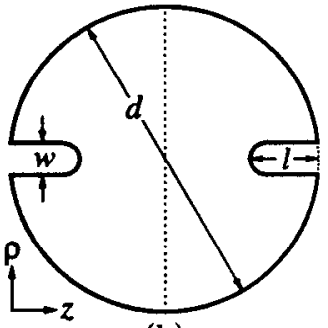

(b)
Figure 1. Outer geometry (a) and cross-section (b) of the bend. The cross-section's dashed line is a symmetry plane.

where $C_{m m}=\beta_{m}$ are the propagation constants and the other $C_{m n}$ involve inner products of the transverse fields.

The power transfer between two modes in a curved section is limited by the difference in their propagation constants. The $\mathrm{TE}_{01}-\mathrm{TM}_{11}$ degeneracy presents a problem, so the degeneracy is split by introducing partial septa perpendicular to the bend plane as shown in Figure $1 \mathrm{~b}$. The modes can no longer be found analytically, but the $\beta_{m}$ can be computed using SUPERFISH[4].

If $\rho_{c} \gg d / 2$ then the coupling is weak and the $T E_{01}-$ like mode amplitude varies little along the bend. A small amount of power will beat in and out of the $n$th coupled mode in an arc length $l_{b} \simeq 2 \pi /\left|\beta_{n}-\beta_{o}\right|$, where $o$ indicates the $\mathrm{TE}_{01}$-like mode. The interaction with each mode cancels when the relative phase advance is a multiple of $2 \pi$. By adjusting the cross-section and $\rho_{c}$, the $\beta$ 's are manipulated so that the three propagating modes coupled to first order all beat out at the end of the $90^{\circ}$ bend.

This is the approach first taken. However, a compact bend which cannot rely on the above assumption is desired. As the coupling coefficients become comparable to the mode spacings, the beat lengths are altered, and modes coupled to second order may be important. The coupling coefficients $C_{m n}$ are required to verify parasitic mode suppression at the end of the bend. Since the $C_{m n}$ are not easily obtained from the field solver, a different approach was taken.

\section{MODES IN CURVED GUIDE}

A curved guide can be treated as a portion of a cylindrically symmetric structure. For the $90^{\circ}$ bend the structure starts at $\phi=0$ and ends at $\phi=\pi / 2$. The fields in the waveguide can be decomposed into modes with azimuthal dependence $e^{i m \phi}$. In the axisymmetric waveguide paradigm the waves propagate along $\phi$ with propagation constant $m$. Compare this with the phase $e^{i \beta z}$ for waves propagating along $z$ with propagation constant $\beta$ in straight waveguides. The curved guide does not close on 
itself so there is no requirement that $m$ be an integer.

The finite element field solver YAP[5] is capable of computing the frequencies of the modes of axisymmetric structures for any real $m$. Non-integral $m$ is allowed. YAP was used to compute dispersion diagrams for curved guide with various cross-sections. One such dispersion diagram is shown in Figure 2. A dispersion diagram for curved guide looks similar to dispersion diagrams for straight guide. However, the simple dispersion formula $\omega^{2} / c^{2}=k_{c}^{2}+\beta^{2}$ for a straight waveguide containing no media does not apply to curved guide. This can be seen best in figure 2 , where the dispersion curves are not parallel lines. A power series of the form

$$
\frac{\omega^{2}}{c^{2}}=k_{c}^{2}+\alpha_{1}\left(\frac{m}{\rho_{c}}\right)^{2}+\alpha_{2}\left(\frac{m}{\rho_{c}}\right)^{4}+\cdots
$$

approximates the dispersion curves well. The cutoff $k_{c}^{2}$ and the coefficients $\alpha_{i}$ depend on $\rho_{c}$ and on the cross-section $\Omega$ of the guide. When $\rho_{c}$ is large then $\alpha_{1} \cong 1$ and the cutoffs $k_{c}^{2}$ are approximately the same between straight and curved guide with the same cross-section. In the large $\rho_{c}$ limit the two approaches described in this paper are equivalent.

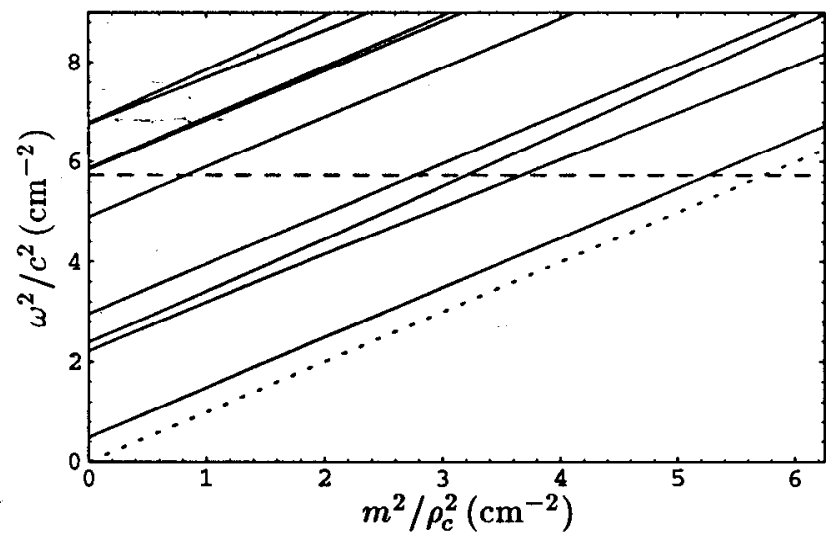

Figure 2. Dispersion diagram of the curved guide for the first design listed in Table 1. The dashed line is the drive frequency $11.424 \mathrm{GHz}$. The dotted line corresponds to the speed of light along the center of the guide.

\section{SCATTERING AT THE INTERFACE}

There is potentially some reflection at the interface between the straight waveguide and the curved waveguide. A generalized scattering matrix $S_{i}$ for the propagating modes in the straight and curved guides can be constructed.

As an example, the scattering matrix for the straightto-curved interface in. an overmoded rectangular H-plane waveguide bend was computed using a mode-matching method. Only $\mathrm{TE}_{n 0}$ modes were considered so the fields are uniform vertically. In the straight guide propagating along $y$ the modes are $E_{z} \propto \sin (2 \pi n x / w)$ where $0 \leq x \leq w$ is the horizontal domain of the waveguide. In the curved guide the modes involve Bessel functions. They are $E_{z} \propto$ $A \mathrm{~J}_{m}(k \rho)+B Y_{m}(k \rho)$ where $\rho_{c}-w / 2 \leq \rho \leq \rho_{c}+w / 2$ and $k=\omega / c$ is the drive frequency. Note that $m$ is real for propagating modes and imaginary for evanescent modes.

The boundary conditions $E_{z}=0$ at $\rho=\rho_{c} \pm w / 2$ yield a characteristic equation for the propagation constants $m$. Solutions were obtained by numerically integrating Bessel's equations and using a shooting method to match the boundary conditions. This yielded numerical values for $m^{2}$ for both propagating $\left(m^{2}>0\right)$ and evanescent $\left(m^{2}<0\right)$ modes. The field $E_{z}$ for each mode was obtained similarly.

The normalized generalized scattering matrix $\mathbf{S}_{\boldsymbol{i}}$ was computed for an example with $w / \lambda=1.36$ and $\rho_{c} / \lambda=$ 3.87 , where $\lambda$ is the free space wavelength. There are two propagating modes in the guides. Using 14 modes for the field expansion on each side of the interface, the computed scattering matrix for the interface is

$$
\mathrm{S}_{i}=\left[\begin{array}{cccc}
4 \cdot 10^{-5} \angle-8^{\circ} & 8 \cdot 10^{-4} \angle-2^{\circ} & 0.982 & 0.190 \\
8 \cdot 10^{-4} \angle-2^{\circ} & 8 \cdot 10^{-4} \angle-122^{\circ} & -0.190 & 0.982 \\
0.982 & -0.190 & 3 \cdot 10^{-4} \angle-4^{0} & 8 \cdot 10^{-4} \angle-4^{\circ} \\
0.190 & 0.982 & 8 \cdot 10^{-4} \angle-4^{\circ} & 8 \cdot 10^{-4} \angle-82^{\circ}
\end{array}\right]
$$

where $\left[a_{s 1}, a_{s 2}, a_{c 1}, a_{c 2}\right]^{T}$ is the incoming wave vector. The wave amplitudes $a_{s n}$ and $a_{c n}$ are for the modes in the straight and curved guides, respectively.

Notice that the reflection amplitude is less than $10^{-3}$. If one assumes the reflections are similar for bends with different cross-sections but similar curvature, then reflection at the straight-to-curved interface can be neglected. The reflected power will be negligible as long as resonances are avoided. The principal concern, then, is mode conversion.

\section{AROUND THE BEND}

The scattering matrix $S_{b}$ for a bend over angle $\phi_{b}$ can be easily computed given $\mathbf{S}_{i}$ for the straight-to-curved interface and the propagation constants $m_{1}$ and $m_{2}$ for the two propagating modes in the curved guide. The example above has $m_{1}=22.85$ and $m_{2}=16.18$. The next mode is evanescent with $m_{3}=i 11.38$. The transmission coefficient for the (straight guide) fundamental mode for various bend angles $\phi_{b}$ was computed. At $\phi_{b}=2 \pi /\left(m_{1}-m_{2}\right)=0.941$ the transmission is nearly perfect. At this bend angle the two propagating waves in the curved guide arrive at the output end of the bend with the same relative phases they had at the input end of the bend. The propagating ficld at the output is the same as at the input except for an overall phase, so waves are faithfully transmitted through the bend with no mode conversion.

The evanescent waves at the interfaces have decayed sufficiently in the curved guide so that they can be neglected in the transmission calculations for $\phi_{b}=0.941$.

This example leads to the principal design criterion for this type of overmoded waveguide bend: the phases $e^{m_{i} \phi_{b}}$ must be identical for all modes propagating in the curved guide. In addition, evanescent modes should be sufficiently above cutoff so that they decay well over the length of the bend, and thus can be neglected. 
Table 1

$90^{\circ}$ Overmoded Waveguide Bends

\begin{tabular}{|c|c|c|c||c|c|c|c|c||c|}
\hline$d(\mathrm{~cm})$ & $l(\mathrm{~cm})$ & $w(\mathrm{~cm})$ & $\rho_{c}(\mathrm{~cm})$ & $m_{1}$ & $m_{2}$ & $m_{3}$ & $m_{4}$ & \multicolumn{1}{|c|}{$m_{5}$} & $f_{c 6}(\mathrm{GHz})$ \\
\hline 4.372 & 0.986 & 0.465 & 31.786 & 72.873 & 60.873 & 56.873 & 52.873 & 28.874 & 11.536 \\
4.275 & 0.971 & 0.611 & 36.655 & 83.867 & 67.867 & 63.867 & 59.867 & 23.868 & 11.819 \\
4.358 & 1.054 & 0.593 & 38.754 & 89.034 & 73.034 & 69.034 & 65.034 & 25.033 & 11.579 \\
3.940 & 0.765 & 0.476 & 23.891 & 53.870 & 41.870 & 37.870 & 33.870 & 9.871 & 12.726 \\
4.157 & 0.904 & 0.622 & 33.894 & 77.212 & 61.212 & 57.212 & 53.212 & 17.213 & 12.163 \\
\hline
\end{tabular}

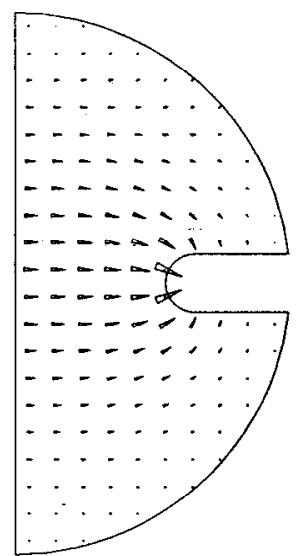

Mode 1 ("TE $\mathrm{TE}_{11}$ ")

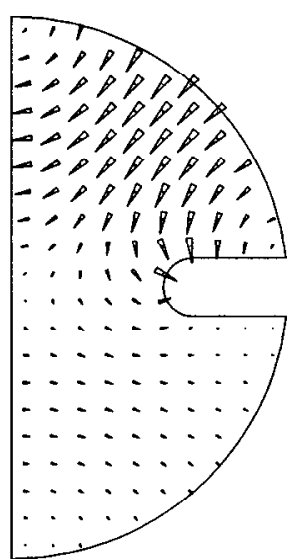

Mode 2 ("TE 21 ")

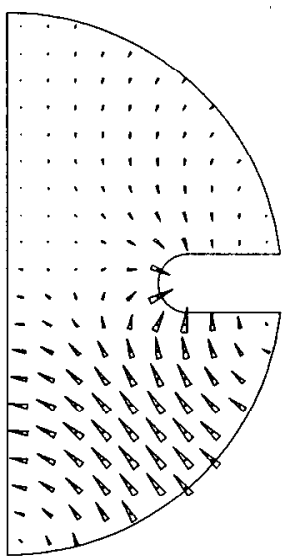

Mode 3 ("TE 31 ")

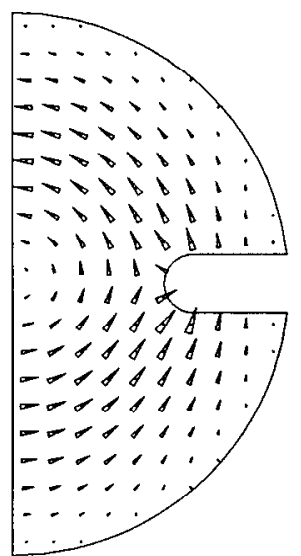

Mode 4 ("TE 01 ")

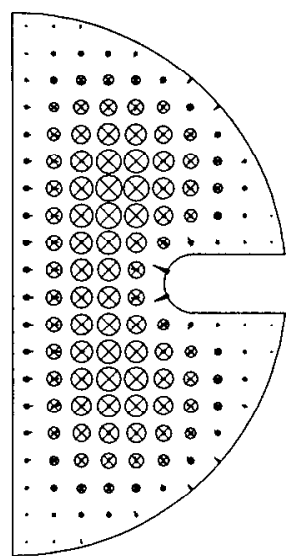

Mode 5 ("TM" $\mathrm{TM}_{11}$ )

Figure 3. Electric field patterns for the five propagating modes of the first design in Table 1.

\section{VI. $90^{\circ}$ BEND DESIGN}

Designs for a $90^{\circ}$ bend with a cross-section as shown in Figure 1b were computed. The phases $e^{m_{i} \pi / 2}$ for the five lowest propagating modes excited by the incoming wave can be fixed relative to each other by adjusting the four parameters: $d, \rho_{c}, l$ and $w$. Propagating modes not excitcd by the incoming wave (due to symmetry) are neglected. Dispersion diagrams were computed using YAP and the bend parameters were adjusted so that the phases were the same. This corresponds to the propagation constants $m_{i}$ differing from one another by multiples of 4 . The cutoff $(m=0)$ frequency of higher order modes were computed in order to discard designs with more than five propagating modes at $11.424 \mathrm{GHz}$. Table 1 lists the parameters for five solutions. It also lists the propagation constants for the five lowest modes and the cutoff frequency $f_{c 6}$ for the sixth lowest mode.

The cross-section in Figure 1 and the dispersion diagrams in Figure 2 correspond to the first design in Table 1. The field patterns for the propagating modes are shown in Figure 3. At cutoff the field patterns for the modes in curved guide are similar to the corresponding modes in straight guide, but for large $m$ the second and third modes are mixed. This is evident in the field plots and in the dispersion diagram, where it appears that the second and third curves are repelling each other. These modes arise, with the introduction of the septa, from the $T E_{21}$ and $T E_{31}$ modes of circular guide. The incoming wave is similar to the fourth mode, which is a $\mathrm{TE}_{01}$-like mode.

The cutoff frequency for the sixth mode of the first design appears close to cutoff. The estimated propagation constant using the straight guide formula is $m_{6} \cong i 10.7$ and the decay amplitude over the length of the waveguide is $e^{i m_{6} \pi / 2}=5 \times 10^{-8}$.

\section{FURTHER WORK}

Further designs can be found, perhaps with smaller radii of curvature and shorter septa so that the bend will have smaller wall losses and be easier to manufacture.

A variation of the YAP field solver will compute the evanescent modes in curved guide. With these modes a mode-matching algorithm can be employed to calculate the scattering matrix $\mathbf{S}_{\boldsymbol{i}}$ for the straight-to-curved guide interface, and then verify that reflections are negligible and that the design criterion is appropriate.

Calculation of the wall losses through the bend and mode-conversion losses (due to manufacturing errors) also requires knowledge of $S_{i}$ in order to obtain the mode amplitudes in the bend as well as the evanescent fields near the interface.

\section{REFERENCES}

[1] R.D. Ruth, "The Development of the Next Linear Collider at SLAC," SLAC-PUB-5729 (1992).

[2] S. A. Schelkunoff, "Generalized Telegraphist's Equations for Waveguides," Bell System Technical Journal, 31, pp. 784-801, July, 1952.

[3] S. P. Morgan, "Theory of Curved Circular Waveguide Containing an Inhomogeneous Dielectric," B.S.T.J., 37, pp. 1209-1251, Sept., 1957.

[4] K. Halbach and R.F. Holsinger, Particle Accelerators 7, 213 (1976).

[5] E.M. Nelson, "A Finite Element Field Solver for Dipole Modes," SLAC-PUB-5881, 1992 Linear Accelerator Conference Proceedings, pp. 814-816. 